\title{
Using Nomograms to Predict the PPCs of Patients With Diffuse Peritonitis Undergoing Emergency Gastrointestinal Surgery
}

\author{
Qiong Xue ${ }^{1 \dagger}$, Yu Zhu ${ }^{1,2 \dagger}$, Ying Wang ${ }^{1}$, Jian-Jun Yang ${ }^{1 *}$ and Cheng-Mao Zhou ${ }^{1 *}$ \\ ${ }^{1}$ Department of Anesthesiology, Pain and Perioperative Medicine, First Affiliated Hospital of Zhengzhou University, \\ Zhengzhou, China, ${ }^{2}$ Department of Scientific Research, Zhaoqing Medical College, Zhaoqing, China
}

\section{OPEN ACCESS}

Edited by:

Marcelo Gama De Abreu,

University Hospital Carl Gustav

Carus, Germany

Reviewed by:

Jian-Guo Zhou,

University of Erlangen

Nuremberg, Germany

Habib Md Reazaul Karim,

All India Institute of Medical Sciences

Raipur, India

*Correspondence:

Jian-Jun Yang

yjyangjj@126.com

Cheng-Mao Zhou

zhouchengmao187@foxmail.com

†These authors have contributed equally to this work

Specialty section:

This article was submitted to

Translational Medicine,

a section of the journal

Frontiers in Medicine

Received: 06 May 2021 Accepted: 24 November 2021 Published: 23 December 2021

Citation:

Xue Q, Zhu Y, Wang Y, Yang J-J and Zhou C-M (2021) Using Nomograms to Predict the PPCs of Patients With

Diffuse Peritonitis Undergoing

Emergency Gastrointestinal Surgery.

Front. Med. 8:705713.

doi: 10.3389/fmed.2021.705713
Objective: To develop and validate a nomogram model for predicting postoperative pulmonary complications (PPCs) in patients with diffuse peritonitis undergoing emergency gastrointestinal surgery.

Methods: We used the least absolute shrinkage and selection operator (LASSO) regression model to analyze the independent risk factors for PPCs in patients with diffuse peritonitis who underwent emergency gastrointestinal surgery. Using R, we developed and validated a nomogram model for predicting PPCs in patients with diffuse peritonitis undergoing emergency gastrointestinal surgery.

Results: The LASSO regression analysis showed that AGE, American Society of Anesthesiologists physical status classification (ASA), DIAGNOSIS, platelets (on the 3rd day after surgery), cholesterol (on the 3rd day after surgery), ALBUMIN (on the first day after surgery), and preoperative ALBUMIN were independent risk factors for PPCs in patients with diffuse peritonitis undergoing emergency gastrointestinal surgery. The area under the curve (AUC) value of the nomogram model in the training group was 0.8240; its accuracy was 0.7000 , and its sensitivity was 0.8658 . This demonstrates that the nomogram has a high prediction value. Also in the test group, the AUC value of the model established by the variables AGE, ASA, and platelets (on the 3rd day after surgery), cholesterol (on the 3rd day after surgery), ALBUMIN (on the first day after surgery), and preoperative ALBUMIN was 0.8240; its accuracy was 0.8000; and its specificity was 0.8986. In the validation group, the same results were obtained. The results of the clinical decision curve show that the benefit rate was also high.

Conclusion: Based on the risk factors AGE, ASA, DIAGNOSIS, platelets (on the 3rd day after surgery), cholesterol (on the 3rd day after surgery), ALBUMIN (on the first day after surgery), and preoperative ALBUMIN, the nomogram model established in this study for predicting PPCs in patients with diffuse peritonitis undergoing emergency gastrointestinal surgery has high accuracy and discrimination.

Keywords: nomogram, PPC, diffuse peritonitis, emergency, gastrointestinal surgery 


\section{INTRODUCTION}

Complicated intra-abdominal infections extend beyond organs and may result in localized or diffused peritonitis (1). Early prognostic assessment and testing for diffuse peritonitis are essential for evaluating disease severity and for providing optimal treatment (2). Postoperative pulmonary complications (PPCs), an important cause of morbidity after upper abdominal surgery, resulting in a significant increase in hospital stays and medical costs (3).

Postoperative pulmonary complications include conditions, such as atelectasis, pleural effusion, pulmonary infection, bronchospasm, acute respiratory failure, and acute respiratory distress syndrome, which are important causes of death and morbidity after major surgery (4).

Therefore, it is of great significance to the prevention and treatment of PPCs to explore risk factors for PPCs after upper abdominal surgery and to screen high-risk patients. These are both hot topics in clinical research. Thus, the individualized prediction of the risk of PPCs is the crux of PPC prevention and treatment. A nomogram model, which integrates relevant risk factors, is able to individually predict adverse clinical events. Therefore, by analyzing the clinical data of patients with diffuse peritonitis undergoing emergency gastrointestinal surgery, this study explores these patients' independent risk factors for PPCs. The aim was to develop and validate individualized prediction of PPCs in patients with diffuse peritonitis undergoing emergency gastrointestinal surgery, assist in the clinical identification of high-risk patients, and provide scientific guidance for the individualized prevention and treatment of PPCs.

\section{MATERIALS AND METHODS}

\section{Patients}

We retrospectively analyzed the medical records of critically ill patients ( $\geq 18$ years old) with diffuse peritonitis who had undergone emergency surgery. Patients with surgery for acute appendicitis, acute cholecystitis, or necrotizing pancreatitis were excluded; patients who had died within the first 7 days after surgery were excluded; and patients with unmeasured total cholesterol levels either before or 1, 3, and 7 days after surgery were excluded as well. Since it was a retrospective study, the requirement for informed consent was waived.

Patient data of the training group and the test group were obtained from BioStudies Database (https://www.ebi.ac.uk/ biostudies/studies/S-EPMC6034864), while patient data of the validation group were obtained from the First Affiliated Hospital of Zhengzhou University.

\section{Perioperative Variables}

The analysis included the following variables: age, sex, body mass index, American Society of Anesthesiologists

Abbreviations: Hb, hemoglobin; HTN, hypertension; DM, diabetes mellitus; CRF, chronic renal failure; ASA, American Society of Anesthesiologists physical status classification; CRP, C-reactive protein; PUL.TBC, pulmonary tuberculosis; -1 , preoperative; 0 , on the day of surgery; 1.1 , on the first day after surgery; 3 , on the 3rd day after surgery. physical status classification (ASA) score, lesion location, diagnosis, perioperative shock, preoperative laboratory findings, postoperative complications, and type of surgery. Pulmonary complications were defined as the presence of one or more of the following postoperative symptoms: pneumonia, atelectasis, pleural effusion, or acute respiratory distress syndrome.

\section{Statistical Analysis}

We used R version 3.1.3 (http://www.R-project) and Empower Stats (http://www.empowerstats.com/cn/download.html) for our analysis. The $\mathrm{R}$ glmnet package was called for least absolute shrinkage and selection operator (LASSO) regression analysis, and lambda was screened through 10 -fold cross-validations. The larger the lambda, the smaller the model. We developed the nomogram prognostic model based on independent prognostic factors and assessed the clinical net benefit by decision curve analysis (DCA). To create each nomogram, we used the RMS package in R. Each individual's total score was calculated according to the nomogram. Then, we performed a diagnostic test on the total score and outcome. We calculated the area under the receiver operating characteristic curve (ROC) curve and its 95\% CI, setting a total score corresponding to the sum of sensitivity and specificity as the cut-off point.

\section{RESULTS}

\section{Analysis of Factors Related to the Prognosis of the Patients}

A total of 1,126 patients were enrolled in this study. The mean age of the PPCs in the training group was $66.2 \pm 13.6$ years, and the mean age of the patients without PPCs was $58.0 \pm 16.5$ years. The characteristics of the remaining baseline are shown in Table 1.

We analyzed the LASSO logistic regression model in the glmnet $\mathrm{R}$ package and determined the optimal $\lambda$ value by cross-validation, with the number of folds set to 10 , as shown in Figures 1A,B. The ordinate represents the target parameter, the lower abscissa represents the $\log (\lambda)$, and the upper abscissa represents the number of non-zero coefficients in the model at this time point. Since it is cross-validation, for each $\lambda$ value, a CI for the target parameter can be obtained around the mean value of the target parameter shown as the red dot. The two dotted lines in Figure 1 represent two special $\lambda$ values, i.e., lambda.min and lambda.1se. lambda.min is the $\lambda$ value of the mean value of the smallest target parameter among the $\lambda$ values. lambda.1se is the $\lambda$ value of the most compact model obtained within a variance range of lambda.min. When lambda.1se is selected as 0.0363 , the variables that enter the model are AGE, ASA, DIAGNOSIS, PLATELET.3, CHOLESTEROL.3, ALBUMIN.1, and ALBUMIN.0. As the $\lambda$ value changes, the model variables are screened, as shown in Figure 1B. Each curve in Figure 1B represents the trajectory of each independent variable coefficient, with the ordinate as the value of the coefficient, the lower abscissa the $\log (\lambda)$, and the upper abscissa the number of non-zero coefficients in the model at this time point. As the 
TABLE 1 | Characteristics of patients in the training, the test, and the validation groups.

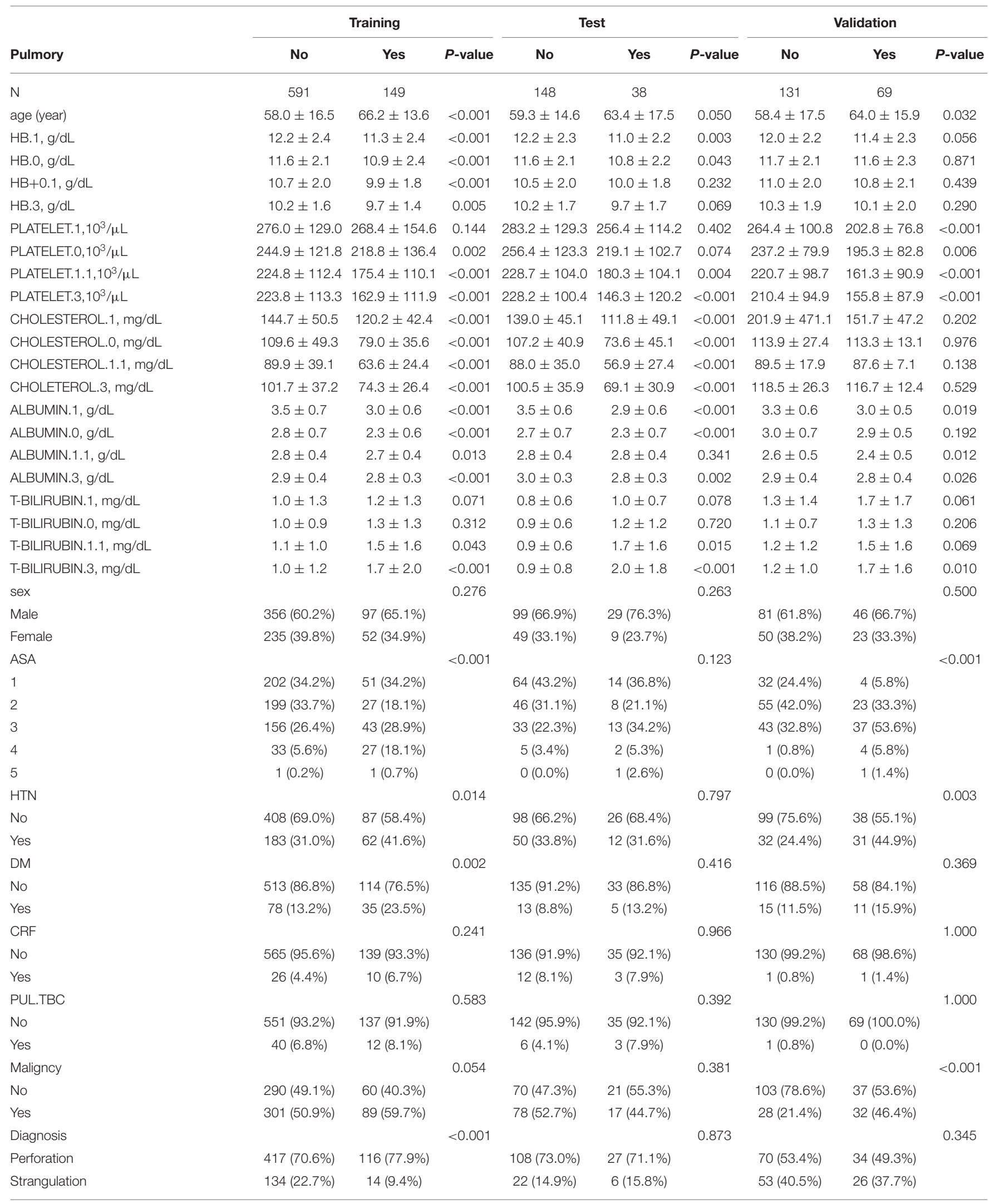


TABLE 1 | Continued

\begin{tabular}{|c|c|c|c|c|c|c|c|c|c|}
\hline Pulmory & \multicolumn{3}{|c|}{ Training } & \multicolumn{3}{|c|}{ Test } & \multicolumn{3}{|c|}{ Validation } \\
\hline Acute mesenteric ischemia & $25(4.2 \%)$ & $11(7.4 \%)$ & & $11(7.4 \%)$ & $4(10.5 \%)$ & & $5(3.8 \%)$ & $7(10.1 \%)$ & \\
\hline Location.of.lesion & & & 0.908 & & & 0.578 & & & 0.342 \\
\hline Stomach & 89 (15.1\%) & $24(16.1 \%)$ & & 25 (16.9\%) & $3(7.9 \%)$ & & 35 (26.7\%) & $20(29.0 \%)$ & \\
\hline Colon and rectum & 258 (43.7\%) & 65 (43.6\%) & & 66 (44.6\%) & 18 (47.4\%) & & 31 (23.7\%) & $11(15.9 \%)$ & \\
\hline Surgery.type & & & 0.051 & & & 0.141 & & & 0.015 \\
\hline Primary repair & $149(25.2 \%)$ & $37(24.8 \%)$ & & $42(28.4 \%)$ & $8(21.1 \%)$ & & $46(35.1 \%)$ & $24(34.8 \%)$ & \\
\hline Small bowel resection with anastomosis & $30(5.1 \%)$ & $10(6.7 \%)$ & & $10(6.8 \%)$ & $0(0.0 \%)$ & & $28(21.4 \%)$ & $7(10.1 \%)$ & \\
\hline Ileo- or jejunostomy & $145(24.5 \%)$ & $28(18.8 \%)$ & & $29(19.6 \%)$ & $11(28.9 \%)$ & & $31(23.7 \%)$ & $30(43.5 \%)$ & \\
\hline
\end{tabular}

Results in the table: Mean + SD/N(\%). Hb, hemoglobin; HTN, hypertension; DM, diabetes mellitus; CRF, chronic renal failure; ASA, American Society of Anesthesiologists physical status classification; CRP, C-reactive protein; PUL.TBC, pulmonary tuberculosis-1, preoperative; 0 , on the day of surgery; 1.1, on the first day after surgery; 3, on the 3rd day after surgery.
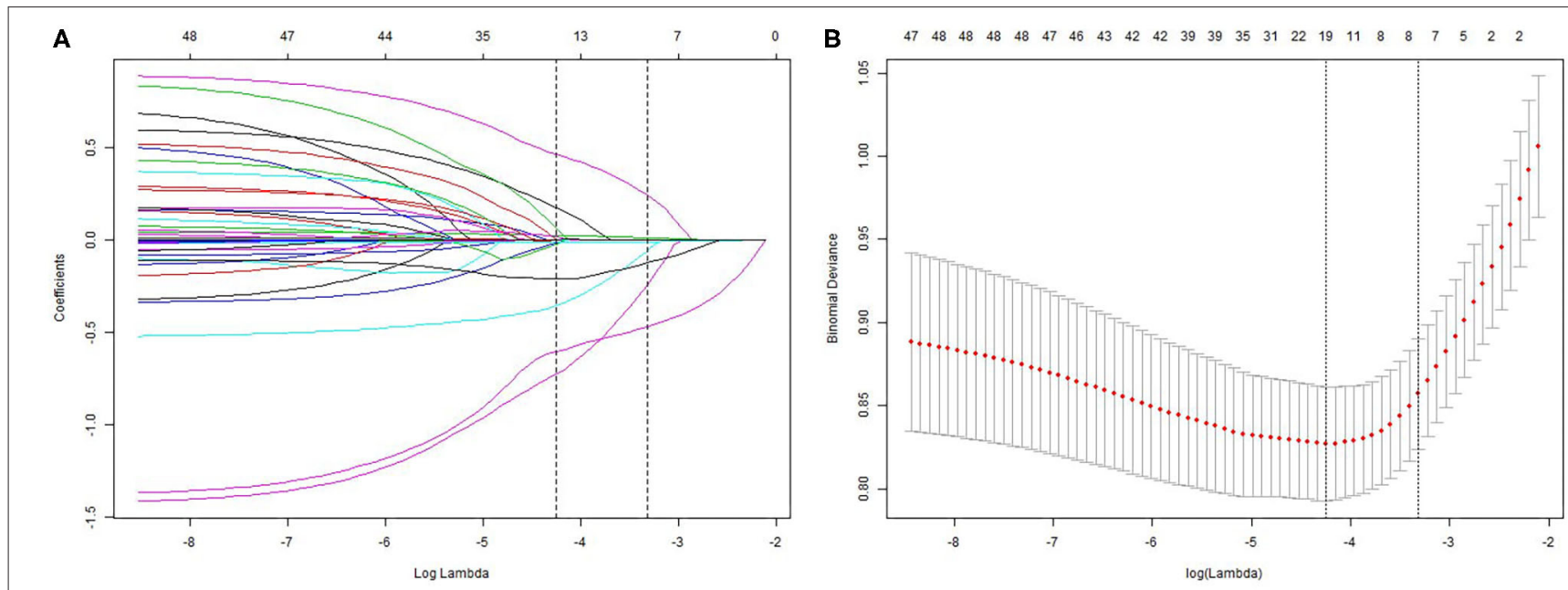

FIGURE 1 | Least absolute shrinkage and selection operator (LASSO) analysis: (A) - Optimal parameter (lambda) selection in the LASSO model; (B) - demographic and clinical feature selection using the LASSO binary logistic regression model.

value of $\lambda$ increases, the degree of compression of the model increases, the number of independent variables entering the model decreases, and the function of the model to select the main variable strengthens.

We based nomogram establishment and evaluation on the independent prognostic factors shown in the logistic regression analysis, and we used $\mathrm{R}$ to establish a nomogram prognostic model for PPCs in patients undergoing emergency gastrointestinal surgery (Figure 2). This incorporated the variables of AGE, ASA, DIAGNOSIS, PLATELET.3, CHOLESTEROL.3, ALBUMIN.1, and ALBUMIN.0. The specific formula is:
Lambda.1se (log) [largest value of lambda such that the error is within $1 \mathrm{SE}$ of the minimum]: $0.0363(-3.3156)$.

Formula for calculating the score (not including the intercept): $0.01263 \times$ AGE $-0.05786 \times$ ASA: $2+0.24632 \times$ ASA: $4-0.24182 \times$ DIAGNOSIS: $2-0.0012 \times$ PLATELET. 3 $0.00896 \times$ CHOLETEROL. 3 - $0.47022 \times$ ALBUMIN.1 - 0.12434 $\times$ ALBUMIN.0.

The total score for the corresponding prediction results could be calculated by adding the scores obtained by projecting the points corresponding to each variable to the "Points" axis. In other words, we calculated the total score based on the score corresponding to each parameter on the nomogram, for predicting each patient's probability of infection. In the 


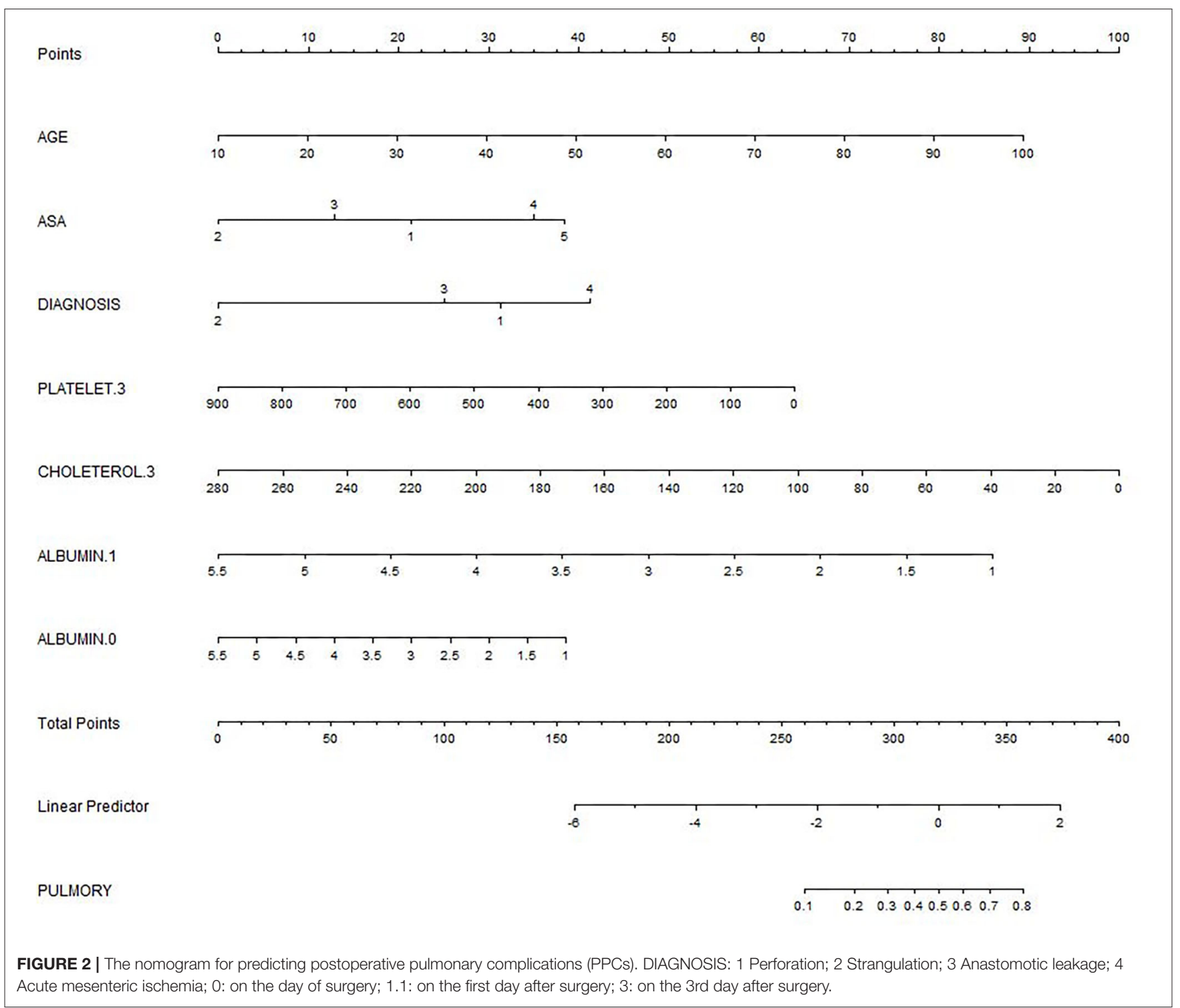

nomogram, we found the score corresponding to the vertical line on the "score" ruler via all the patients' variable values. We collected the scores of all the variable values and then found the points corresponding to the vertical line of the "predictive ruler" in the cumulative "total score" ruler. According to the score on the predictive ruler, we obtained the corresponding probability from the "incidence of PPCs" ruler, which was the patients' probability of PPCs. The area under the curve (AUC) value of the nomogram model in the training group was 0.8240 , the accuracy was 0.7000 , and the sensitivity was 0.8658 , demonstrating that the nomogram had a higher predictive value. Also in the test group, the AUC value of the nomogram model by the variables of AGE, ASA, DIAGNOSIS, PLATELET.3, CHOLESTEROL.3, ALBUMIN.1, and ALBUMIN.0 was 0.8240, the accuracy was 0.8495 , and the sensitivity was 0.8986 . We also validated the results of these studies on the test dataset and the validation group dataset (Shown in Figure 3, Table 2).

The DCA evaluated the nomogram prognostic model with higher net income for different decision thresholds. Figure 4 shows DCA analysis in the modeled population. For example, the abscissa in Figure 4 represents the threshold probability: in the risk assessment tool, the probability of patients being diagnosed with PPCs is recorded as $\mathrm{Pi}$; when $\mathrm{Pi}$ reaches a threshold (denoted as Pt), it is defined as positive, and treatment measures are taken. At this time, there will be benefits (advantages) for patient treatment, non-patient treatment injuries, and untreated losses (disadvantages); the ordinate is the net benefit after the reduction. The results of this study show that the model has a high benefit rate for patients. We also validated the results of these studies on the test dataset and the validation group dataset. 

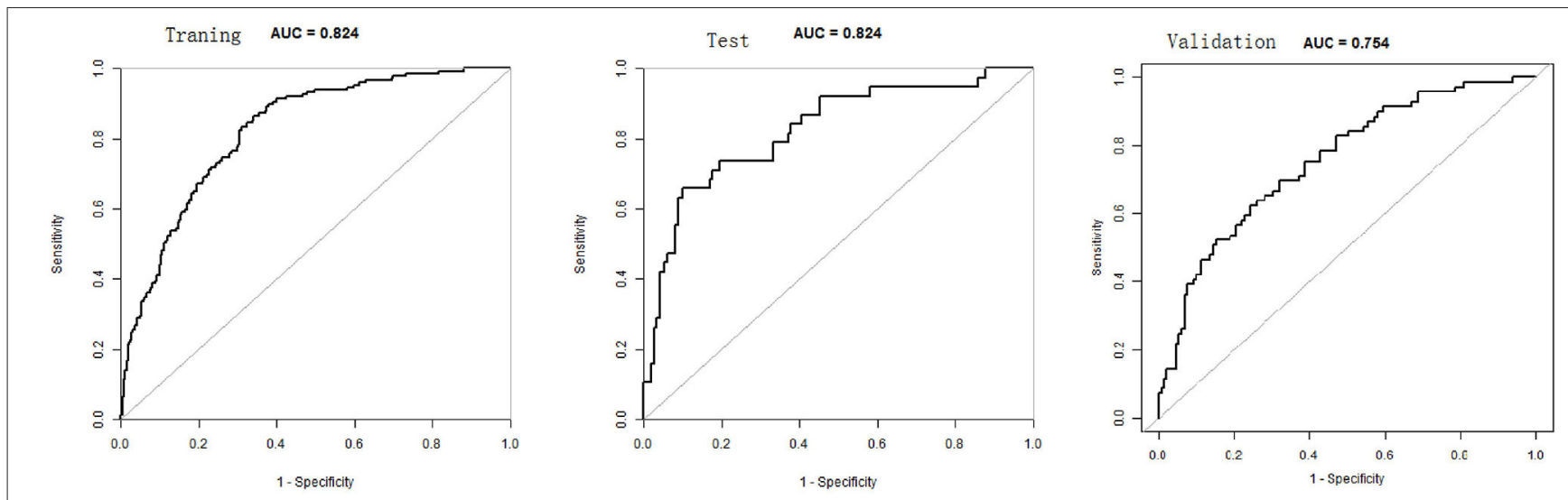

FIGURE 3 | The receiver operating characteristic curve (ROC) for each nomogram.

TABLE 2 | Nomogram model for evaluating the training, the test, and the validation groups.

\begin{tabular}{lccc}
\hline & Traning & Test & Validation \\
\hline ROC area (AUC) & 0.8240 & 0.8240 & 0.7535 \\
$95 \% \mathrm{Cl}$ low & 0.7896 & 0.7452 & 0.6837 \\
$95 \% \mathrm{Cl}$ upp & 0.8585 & 0.9027 & 0.8233 \\
Best threshold & -1.7099 & -0.5823 & -0.4386 \\
Specificity & 0.6582 & 0.8986 & 0.7557 \\
Sensitivity & 0.8658 & 0.6579 & 0.6232 \\
Accuracy & 0.7000 & 0.8495 & 0.7100 \\
Positive-LR & 2.5330 & 6.4912 & 2.5512 \\
Negative-LR & 0.2039 & 0.3807 & 0.4986 \\
Diagnose-OR & 12.4210 & 17.0513 & 5.1166 \\
N-for-diagnose & 1.9085 & 1.7968 & 2.6391 \\
Postive-pv & 0.3897 & 0.6250 & 0.5733 \\
Negative-pv & 0.9511 & 0.9110 & 0.7920 \\
\hline
\end{tabular}

Positive PV, positive predictive value; Negative PV, negative predictive value; Accuracy, positive - $L R$, positive likelihood ratio; Negative - $L R$, negative likelihood ratio; Diagnostic $O R$, diagnostic ratio; Number for Diagnostics, number of tests required for diagnosis.

\section{DISCUSSION}

Postoperative pulmonary complications s after major surgery are an important cause of death and morbidity (4). The hospitalization time for patients at high risk for PPCs is also significantly longer than that of low-risk patients (5). A recent large-scale prospective observational study has shown that in patients who had undergone non-cardiac surgeries under general anesthesia with muscle relaxants, the incidence of PPCs was 7.6\%. High-risk surgeries include intrathoracic surgery and upper abdominal open surgery. Prediction and early identification of high-risk patients with PPCs assume important clinical significance by helping clinicians take proactive measures and provide timely treatment. In this study, we analyzed clinical data from patients with diffuse peritonitis undergoing emergency gastrointestinal surgery using the LASSO regression. We found that AGE, ASA, DIAGNOSIS, PLATELET.3, CHOLESTEROL.3,
ALBUMIN.1, and ALBUMIN.0 were independent risk factors for PPCs in patients with diffuse peritonitis.

Quantifying individualized PPC prediction in patients with diffuse peritonitis undergoing emergency gastrointestinal surgery remains a knotty medical problem at present. The nomogram model can quantify and visualize logistic regression results to individualize predictions of the risk of adverse clinical events. This study was based on independent PPC risk factors, such as AGE, ASA, DIAGNOSIS, PLATELET.3, CHOLESTEROL.3, ALBUMIN.1, and ALBUMIN.0, in patients with diffuse peritonitis. We established a nomogram model. The results showed that the AUC value of the nomogram model for the training group was 0.8240 , the accuracy was 0.7000 , and the sensitivity was 0.8658 . This indicates that the nomogram had a higher predictive value. Also in the test group, the AUC value was 0.8240 , the accuracy was 0.8 , and the specificity was 0.8986 . The results of the calibration curve and the clinical decision curve evaluation models were also good.

The preoperative factors age and ASA classification were independent risk factors for PPCs in these patients, and this was consistent with the results reported in several previous studies (6-8). The results of this study indicated that ASA scores III and IV increased the weight of the nomogram model score by approximately 13 and 35 , respectively.

Moreover, studies have shown that preoperative Platelet to Lymphocyte Ratio (PLR) and Neutrophil to Lymphocyte Ratio (NLR) are good prognostic factors for PPCs and overall survival (OS) in patients with Nonsmall-Cell Lung Cancer (NSCLC) undergoing radical lung cancer surgery (9). In this study, we also found that a platelet value of 200 on the third day would increase the weight of the nomogram model score by 50 .

High-density lipoprotein cholesterol (HDL-C) levels appear to be one of the risk factors for postoperative nosocomial infections (10). Studies (11) have also shown that a decrease in serum cholesterol levels is a poor prognostic factor in patients with severe community-acquired pneumonia. In this study, we also found that cholesterol levels were associated with PPCs. The cholesterol value being 100 on the third day would increase the weight of the nomogram model score by approximately 62 . 

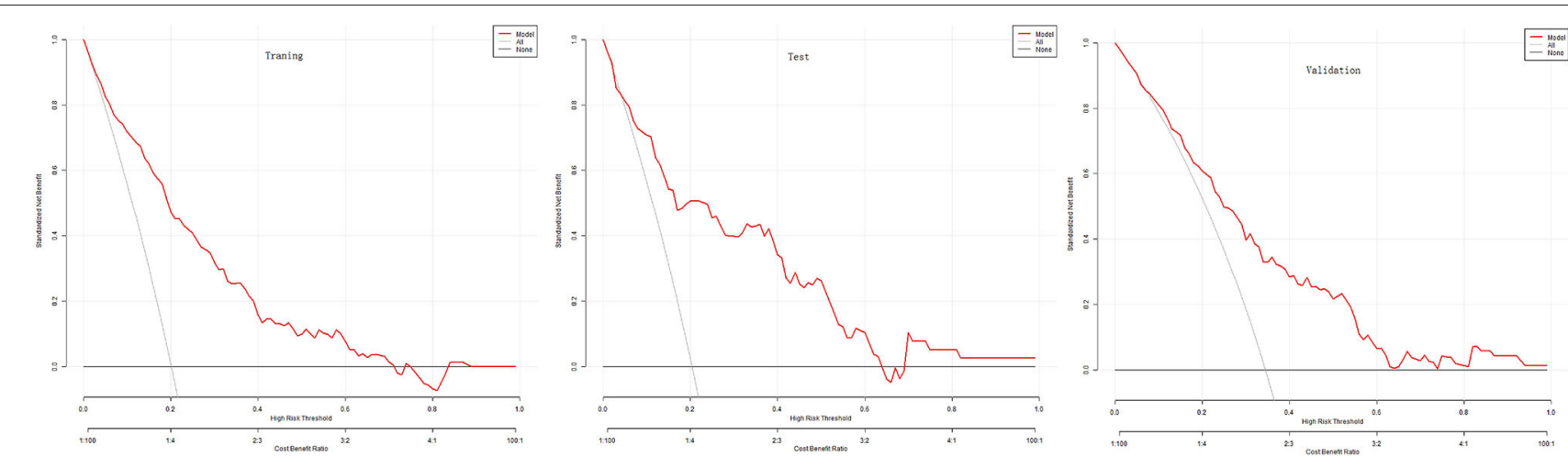

FIGURE 4 | Decision curves for each nomogram.

Moreover, studies (12) have shown that perioperative serum albumin changes are predictive factors of PPCs in patients with lung cancer. Similarly, preoperative albumin levels can be used as predictive factors of PPCs after elective laparoscopic gastrectomy (13). In our study, we also found that albumin levels were associated with PPCs. An albumin value of 1.5 on the first day would increase the weight of the nomogram model score by approximately 78 .

Although we have included several prognostic factors to establish a more accurate prediction of postoperative survival in patients with non-metastatic bowel cancer, there remain several limitations to this study. First, there was only one source for the sample. Since there was only one disease involved in this study, the model for the overall population needs further improvement. Second, in this study, we use internal verification. Therefore, the findings are not necessarily applicable to external data. Third, this study is retrospective, so there may have been selection bias in the data collection. Finally, PPC diagnosis is dependent on clinical judgment and confirmed by imaging and laboratory examination. Therefore, some lung infections, pleural effusion, and atelectasis that do not show obvious symptoms may be overlooked, leading to an underestimation of PPC incidence.

\section{CONCLUSION}

Our research results showed that AGE, ASA, platelets, cholesterol, and ALBUMIN are high-risk factors for pulmonary complications after gastrointestinal emergency surgery in patients with peritonitis. Moreover, the nomogram established by the above factors can quantitatively evaluate the likelihood of pulmonary complications.

\section{REFERENCES}

1. Sartelli M, Catena F, Ansaloni L, Moore E, Malangoni M, Velmahos G, et al. Complicated intra-abdominal infections in a worldwide context: an observational prospective study (CIAOW Study). World J Emerg Surg. (2013) 8:1-7. doi: 10.1186/1749-7922-8-1

2. Sartelli M. A focus on intra-abdominal infection. World J Emerg Surg. (2010) 5:9. doi: 10.1186/1749-7922-5-9

\section{DATA AVAILABILITY STATEMENT}

Publicly available datasets were analyzed in this study. This data can be found here: Data is available at BioStudies database (https://www.ebi.ac.uk/biostudies/studies/S-EPMC6034864), accession numbers: S-EPMC6034864.

\section{ETHICS STATEMENT}

The studies involving human participants were reviewed and approved by the Ethics Committee at the First Affiliated Hospital of Zhengzhou University (2021-KY-677). Because it was a retrospective study, the Ethics Committee exempted patient informed consent. Written informed consent for participation was not required for this study in accordance with the national legislation and the institutional requirements.

\section{AUTHOR CONTRIBUTIONS}

All authors contributed to the article, data analysis, drafting or revising of the article, final approval of the version for publication, agreed to be accountable for all aspects of the work, prepared Figures 1-4, and approved the submitted version.

\section{ACKNOWLEDGMENTS}

We are grateful to Prof. Hwan for disclosing data (14) and like to acknowledge a debt of gratitude to the public BioStudies Database, and also thank Duan Wen for helping us deal with the pictures together. 
a systematic review and meta-analysis. Lancet Respir Med. (2014) 2:1007-15. doi: 10.1016/S2213-2600(14)70228-0

5. Neto AS, da Costa LG, Hemmes SN, Canet J, Hedenstierna G, Jaber $\mathrm{S}$, et al. The LAS VEGAS risk score for prediction of postoperative pulmonary complications. Eur J Anaesthesiol. (2018) 35:691. doi: 10.1097/EJA.0000000000000845

6. Ogawa F, Satoh Y, Iyoda A, et al. Clinical impact of lung age on postoperative readmission in non-small cell lung cancer. J Surg Res. (2015) 193:442-8. doi: $10.1016 /$ j.jss.2014.08.028

7. Silva D R, Gazzana M B, Knorst M M. Merit of preoperative clinical findings and functional pulmonary evaluation as predictors of postoperative pulmonary complications. Rev Associação Méd Brasil. (2010) 56:551-7. doi: 10.1590/S0104-42302010000500016

8. Wang S, Li X, Li Y, Li J, Jiang G, Liu J, et al. The long-term impact of postoperative pulmonary complications after video-assisted thoracic surgery lobectomy for lung cancer. J Thorac Dis. (2017) 9:5143-52. doi: 10.21037/jtd.2017.10.144

9. Lan H, Zhou L, Chi D, Zhou Q, Tang X, Zhu D, et al. Preoperative platelet to lymphocyte and neutrophil to lymphocyte ratios are independent prognostic factors for patients undergoing lung cancer radical surgery: a single institutional cohort study. Oncotarget. (2017) 8:35301-10. doi: 10.18632/oncotarget.13312

10. Canturk NZ, Canturk Z, Okay E, Yirmibesoglu O, Eraldemir B. Risk of nosocomial infections and effects of total cholesterol, HDL cholesterol in surgical patients. Clinical Nutrition. (2002) 21:431-6. doi: $10.1054 /$ clnu.2002.0575

11. Chien YF, Chen CY, Hsu CL, Chen KY Yu CJ. Decreased serum level of lipoprotein cholesterol is a poor prognostic factor for patients with severe community-acquired pneumonia that required intensive care unit admission. J Crit Care. (2015) 30:506-10. doi: 10.1016/j.jcrc.2015. 01.001
12. Li P, Li J, Lai Y, Wang Y, Wang X, Su J, et al. Perioperative changes of serum albumin are a predictor of postoperative pulmonary complications in lung cancer patients: a retrospective cohort study. J Thorac Dis. (2018) 10:5755-63. doi: $10.21037 /$ jtd.2018.09.113

13. Chen $\mathrm{Y}, \mathrm{Wu}$ G, Wang R, Chen J. Preoperative albumin level serves as a predictor for postoperative pulmonary complications following elective laparoscopic gastrectomy. Curr Pharm Des. (2018) 24:3250-5. doi: 10.2174/1381612824666180713104307

14. Lee SH, Lee JY, Hong TH, Kim BO, Lee YJ, Lee JG. Severe persistent hypocholesterolemia after emergency gastrointestinal surgery predicts inhospital mortality in critically ill patients with diffuse peritonitis. PLOS ONE. (2018) 13:e0200187. doi: 10.1371/journal.pone.0200187

Conflict of Interest: The authors declare that the research was conducted in the absence of any commercial or financial relationships that could be construed as a potential conflict of interest.

Publisher's Note: All claims expressed in this article are solely those of the authors and do not necessarily represent those of their affiliated organizations, or those of the publisher, the editors and the reviewers. Any product that may be evaluated in this article, or claim that may be made by its manufacturer, is not guaranteed or endorsed by the publisher.

Copyright (c) 2021 Xue, Zhu, Wang, Yang and Zhou. This is an open-access article distributed under the terms of the Creative Commons Attribution License (CC BY). The use, distribution or reproduction in other forums is permitted, provided the original author(s) and the copyright owner(s) are credited and that the original publication in this journal is cited, in accordance with accepted academic practice. No use, distribution or reproduction is permitted which does not comply with these terms. 